\title{
SOME DESIGNS AND APPLICATIONS FOR PAGKAGED AMPLIFIERS USING SUBMINIATURE TUBES
}

B. ChANCE, J. N. ThURSTON, P. L. RICHMAN

TECHNICAL REPORT NO. 20

\section{RESEARCH LABORATORY OF ELECTRONICS}

MASSACHUSETTS INSTITUTE OF TECHNOLOGY 


\title{
Some Designs and Applications for Packaged Amplifiers Using Subminiature Tubes*
}

\author{
Britton Chance, ** J. N. Thurston, and P. L. Richman \\ Research Laboratory of Electronics, Massachusetts Institute of Technology, Cambridge, Massachusetts
}

(Received December 12, 1946)

\begin{abstract}
Design and construction details of d.c. and a.c. amplifier packages using subminiature tubes are given. In addition, applications to control and measurement circuits are shown. The purpose of these amplifiers is to decrease the time lost in the construction and repair of complicated electronic equipment for biophysical and other experiments.
\end{abstract}

\section{INTRODUCTION}

$T^{\prime}$ HE employment of subminiature vacuum tubes in packaged assemblies is well known in hearing aids, proximity fuses, ${ }^{1}$ and small radios. ${ }^{2,3}$ The use of the indirectly heated types in functional sub-assemblies was begun as part of a program to decrease the weight and to in-

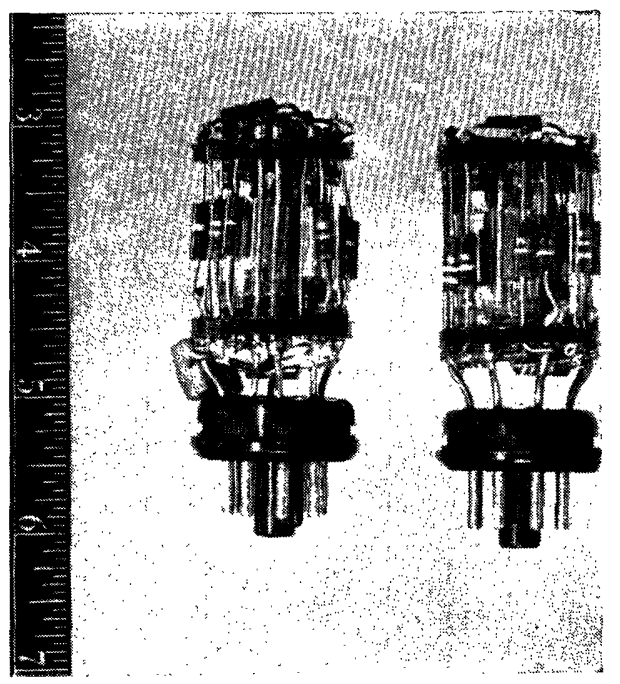

FIG. 1. Packaged amplifiers using subminiature tubes. On the left is an a.c. amplifier of gain of 1000 with feedback, while on the right is a d.c. amplifier of gain of 1000 without feedback.

* The research reported in this paper was made possible in part through support extended the Massachusetts Institute of Technology, Research Laboratory of Electronics, jointly by the Army Signal Corps, the Navy Department (Office of Naval Research), and the Army Air Forces (Air Material Command), under the Signal Corps Contract No. W-36-039 sc-32037.

** John Simon Guggenheim Memorial Fellow, now at the Medical Nobelinstitutet, Stockholm, Sweden.

1 F. Rockett, "Proximity fuse," Electronics 18 [11], 110-111 (1945).

${ }^{2}$ C. Brunetti and A. S. Khouri, "Printed electronic circuits," Electronics 19 [4] 104, (1946).

3 F. Rockett, "Midget electronic equipment," Electronics 20, 84 (1947). crease the serviceability of a Loran indicator ${ }^{4}$ and an aircraft navigational computer. ${ }^{5}$

In each one of the previous cases the circuit and package designs were directed towards specific military or commercial objectives. The object of the present work was to determine whether the principles of compact packaging and easy serviceability could be applied to the design of laboratory equipment. Many, many hours are lost by inexpert circuit work of chemists, physicists, and biologists. Often important data are lost because of the failure of equipment during a critical experiment. Particularly in the study of enzyme kinetics where many weeks may be spent in purifying a fragile protein, an apparatus failure may be disastrous. In such cases the faulty amplifier must be replaced immediately; there is no time to determine which tube or soldered joint may have failed. A year's experience with packaged amplifiers used in sensitive spectrophotometry has convinced the author that they greatly simplify the construction and maintenance of electronic equipment.

In approaching this problem one may ask what the advantages of such a technique are. Some obvious ones are listed below.

(1) Ease and speed in design and construction of complex circuits.

(2) Standardization of all units to the same performance, in spite of variations in tube and component characteristics.

(3) Ease of replacement of functional units in case of failure with subsequent discard or factory repair of faulty units.

(4) Greatly decreased parts and labor costs in apparatus assembly.

\footnotetext{
${ }^{4}$ Radiation Laboratory Series (McGraw-Hill Book Company, Inc.), 20, Chapter 7 (in press).

${ }_{5}$ Radiation Laboratory Series (McGraw-Hill Book Company, Inc.), 21 (in press).
} 


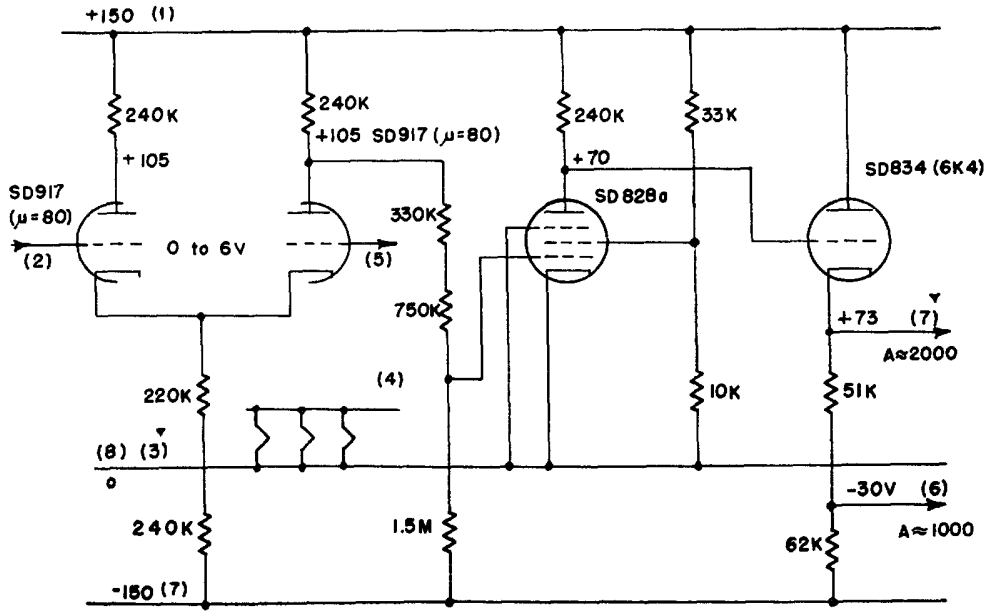

(a)

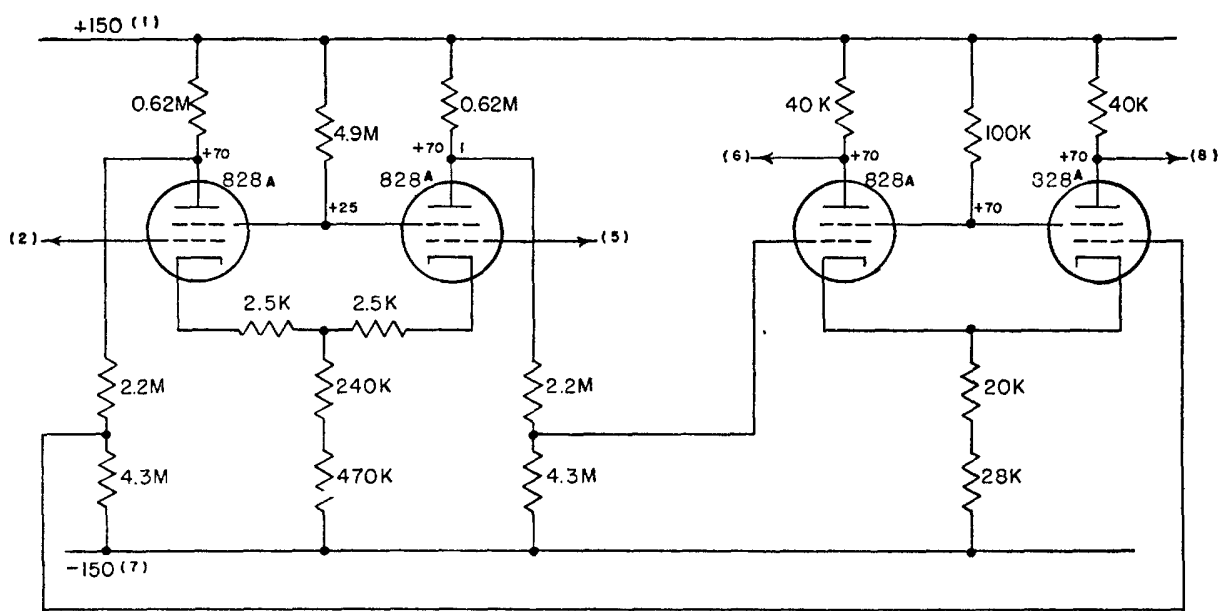

(b)

FIG. 2. Circuit diagram of two designs of d.c. amplifiers. That of Fig. 2a has a single-ended cathode-follower output, while that of Fig. $2 \mathrm{~b}$ has push-pull output. The former is more sensitive to supply voltage changes. The gain of each unit is roughly 1000 . The numbers refer to pins on the octal base in this and in subsequent figures.

A useful packaged unit should have the following qualities :

(1) An adequate tube life (a few thousand hours).

(2) A design flexible enough to perform a number of functions.

(3) The high performance required for physical measurements (low noise, microphonics, and grid current; high gain; adequate band width; low output impedance, etc.).

In view of these factors it was decided to construct two basic types of amplifiers, direct and capacitance-coupled, each suitable for large voltage or current ${ }^{6}$ amplification. Furthermore, the

${ }^{6}$ In conjunction with a suitable output tube (see Figs. 3 and 4 ). package was to be mounted on a standard octal base for easy interchangeability.

Subminiature tubes having indirectly heated cathodes are available from Raytheon and Sylvania and the $T-3$ types made by the latter company are used in these circuits. The types include a low $-\mu$ triode $6 \mathrm{~K} 4$ or SD834, similar to type $6 \mathrm{~J} 5$; a high- $\mu$ triode SD-917A, similar to type 6SL7 (one section only); and a pentode SD828A, roughly equivalent to type $6 \mathrm{SJ} 7$. Although these tubes are rated for somewhat higher plate voltages, these designs have employed $150 \mathrm{v}$ in order to minimize resistor dissipation and thereby to permit the use of $\frac{1}{2}$ watt 
or smaller resistors. Complete specifications on these tubes are now, or shortly will be, available from the manufacturer. Some experimentally determined characteristics of these tubes are given under the discussion of amplifier performance.

Figure 1 shows a type of construction used in laboratory models. An octal plug is used for the base and wire uprights and bakelite spacers are used for mounting the tubes and components. Components requiring adjustment for standardizing gain or quiescent potentials are accessible on top of the unit. The left amplifier is capacitance-coupled and has three tubes, while the one on the right has four tubes and is directcoupled. The latter represents a reasonable limit of complexity for the octal base, and if more tubes or components are required for a particular function, the eleven-pin base is used. ${ }^{7}$ It is felt, however, that three or four tubes and 10 to 20 components make a package of reasonable complexity.

\section{DIRECT-COUPLED AMPLIFIERS}

\subsection{Design and Performance}

Two circuits for direct-coupled amplifiers have been designed and tested and are shown in Fig. 2. The circuit of Fig. 2a has considerable gain due to the pentode, and a low output impedance as a result of the cathode follower. The input amplifier depends upon similarity of the variation of contact potential of the input grids for stabilization against heater voltage fluctuations and aging. The large cathode resistor also permits subtraction of two independent input signals - a very useful feature in feed-back circuits. Feedback is not included in this circuit, since the loop is usually closed through a current-carrying tube (see Figs. 3 and 4).

A serious limitation to the stability of this circuit is its sensitivity to line-voltage fluctuations, not at the input stage, but at the grid of the pentode. Fluctuations of the negative supply are reduced by a factor of but $1 / 15$ at this point whereas the large common cathode resistor makes the effect of negative supply variations negligible at the input grids.

This difficulty is avoided by a well-regulated supply (see Fig. 3) or by the circuit of Fig. 2b. In the latter circuit the second amplifier is also differential, and for similar tubes and components voltage variations affect only the common and not the differential output voltage. The output resistance of the amplifier is, however, much higher than that of Fig. 2a, and some care must be taken with the output coupling networks. This circuit has another useful feature; the separate cathode resistors of the first stage per-

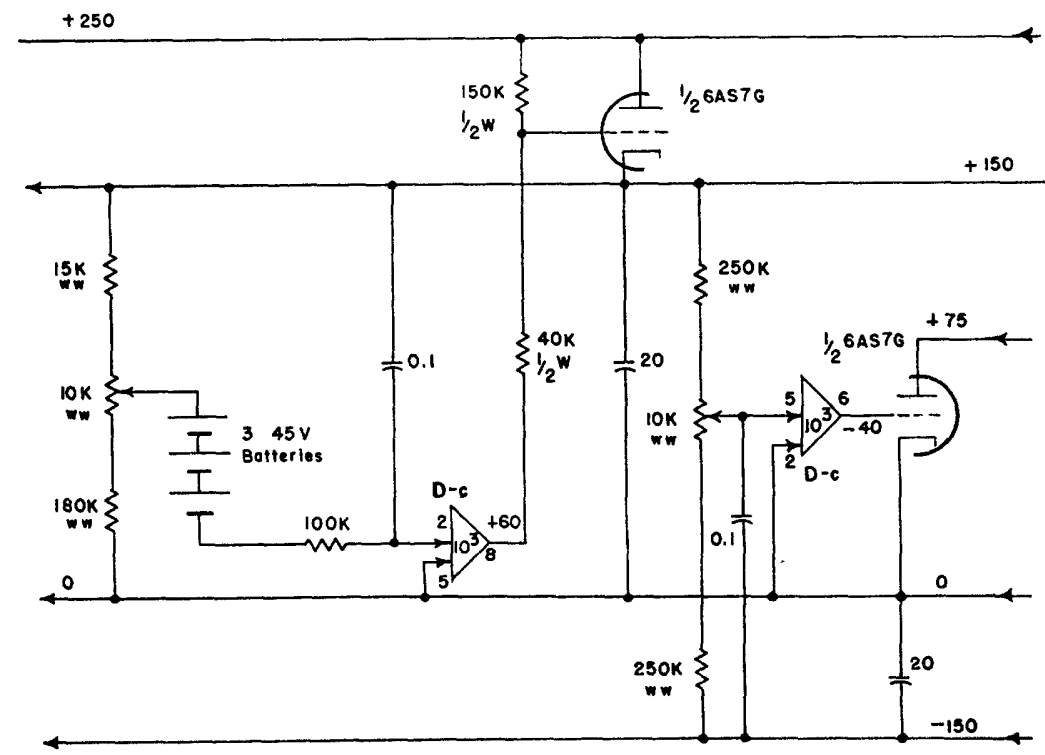

FIG. 3. Voltage stabilizer using packaged subminiature d.c. amplifiers and type 6AS7G control tubes. The d.c. amplifiers are indicated by the triangles. The input voltages are between 0 and +250 , and -150 and +75 ; the output is $-150-0-+150$.

${ }^{7}$ This socket was the basis of several Radiation Laboratory designs by R. M. Walker. 
mit a bias adjustment so that zero differential output is obtained for equal input voltages.

The gain of these two amplifiers is between 600 and 1200 but is usually maintained constant by a feed-back network as shown in Fig. 4. Some tube selection permits a grid current of $0.01 \mu \mathrm{a}$ or less. The drift rate of these amplifiers has not been completely determined, and the use of other techniques is recommended where a drift rate of less than $20 \mu \mathrm{v} / \mathrm{min}$. is required. Type 6SU7/ GTY may be used as a preamplifier, or, for frequencies up to 20 c.p.s., the modulator-demodulator circuit described later may be used. Noise and microphonics are similar to that obtained from the a.c. amplifiers.

\subsection{Applications}

Many examples of the application of these packaged amplifiers to typical circuit designs are given in a companion paper ${ }^{8}$ and two are shown here in Figs. 3 and 4 . In the first example is shown a voltage stabilizer for these amplifiers. There are two separate stabilizers; one for the positive and one for the negative bus. The 135-v reference battery was chosen in order to minimize the effects of drift in the d.c. amplifier. This voltage is subtracted from an adjustable portion (10K potentiometer) of the output voltage resulting in a quiescent potential of roughly zero at the grid (pin 2) of the d.c. amplifier. The other grid of the d.c. amplifier is grounded; thereby the remainder from the subtraction of this portion of the output voltage and the reference voltage is equated to zero. The control tube has a plate voltage of roughly 100 volts and requires a quiescent bias of about $-40 \mathrm{v}$ for 75 -ma loads. The proper bias is obtained from a coupling network ( $40 \mathrm{~K}$ and $150 \mathrm{~K})$. The characteristics of the type 6AS7G tube permit rather large output currents at this plate voltage. The negative supply stabilizer uses the whole positive supply as a reference voltage and equates the difference of the two to zero. Here a "floating" supply for the control tube (6AS7-G) is used; one end is connected to $-150 \mathrm{v}$, the other supplies a plate voltage of $75 \mathrm{v}$ for the control tube.

The heaters of the d.c. amplifiers are preferably operated from d.c., or from regulated a.c. A

${ }^{8}$ B. Chance, "Stable spectrophotometry of small density changes," Rev. Sci. Inst. 18, 601 (1947).

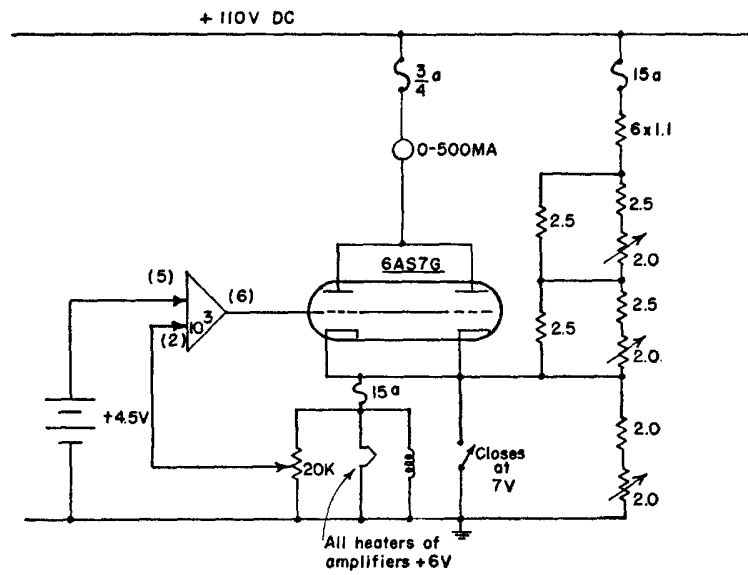

FIG. 4. Heater voltage stabilizer. A d.c. amplifier and a $6 \mathrm{AS7}-\mathrm{G}$ regulate $6.0 \mathrm{v}$ at $10 \mathrm{amp}$. with reference to a $4.5 \mathrm{v}$ battery. A protection circuit is included.

simple regulator capable of supplying $10 \mathrm{amp}$. at $6 \mathrm{v}$ d.c., is indicated in Fig. 4. Here a 4.5-v reference voltage is equated to a portion of the output voltage obtained from the $20 \mathrm{~K}$ potentiometer. The amplified error voltage varies the portion of the output current carried by the 6AS7-G, usually operated with a quiescent current of $125 \mathrm{ma}$ per section. Better performance is readily obtainable by using a second amplifier unit and, if necessary, a type 6SU7/GTY preamplifier. To avoid overvoltage if some amplifier units are removed, a relay is adjusted to short circuit the output intermittently when the output exceeds $7 \mathrm{v}$. This regulator depends upon a $110-v$ d.c. power line and wastes considerable power in the voltage-dividing network. It is possible that control of an a.c. line followed by copper-oxide rectifiers and filters would be more economical.

Another application of the d.c. amplifier is shown in a companion paper ${ }^{8}$ (Fig. 3), illustrating its use in an oscillograph driver. Here the variable input is equated to the output from the 6AS7-G. The result is linear operation and low output resistance of the driver unit over a range of $\pm 50 \mathrm{ma}$ with a 62 -ohm cathode resistor, and up to \pm 100 ma with smaller values. The low resistance $6 \mathrm{v}$ heater supply is used for bucking out the quiescent current through the oscillograph. The limits of operation of the oscillograph are set by the diode-connected triodes. This setting is, however, affected by the $110-v$ d.c. 
line since the diodes limit potentials within the feed-back loop. In cases where variations of the diode contact potential are not important, they may be used to limit the amplitude of the input signal and thereby avoid this difficulty.

\section{A.C. AMPLIFIERS}

\subsection{Circuit Designs}

The circuits for triode and pentode amplifiers are shown in Figs. 5a and 5b, respectively. Both circuits employ fixed bias for the cathodes (and screens) and have cathode-follower output. Since these amplifiers are of ten used directly for amplitude measurement, internal negative feedback is provided to permit standardization of gain. The open-loop gain of the pentode unit is roughly $10^{4}$, and the closed-loop gain of $10^{3}$ is reasonably independent of drifts in tube characteristics. The gain of the triode unit is but $4 \times 10^{3}$ for high- $\mu$ triodes $(\mu=80-90$, SD917) and provides

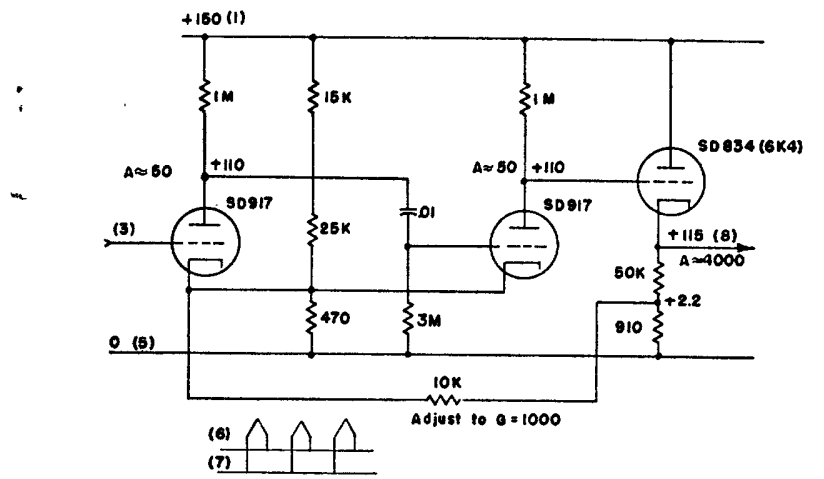

(a)

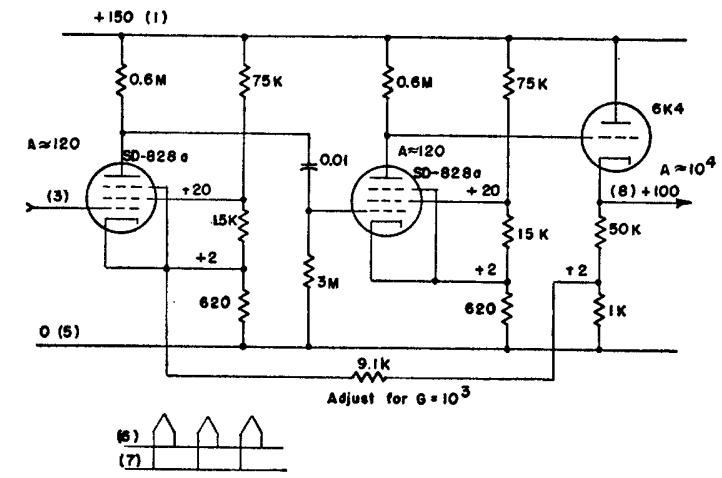

(b)

FIG. 5. Circuit diagrams for two designs of a.c. amplifiers, one using triodes (5a) and one using pentodes (5b). The gain figures for the triodes are for Type SD-917 $(\mu=80)$. The closed-loop gain of both amplifiers is adjusted to 1000 . barely adequate constancy of gain. With presently available triodes $(\mu=40$, SD917A) the closed-loop gain would have to be reduced to about 200 to secure comparable stability. The common cathode resistor causes some positive feedback between the two amplifier stages. A complete discussion of the properties of such circuits involving positive and negative feedback is given in Radiation Laboratory Series Vol. 18, Section 11.13 .

The low frequency response is set by the single coupling network in the amplifier $(R C=0.03$ sec.) although space is available for a compact condenser to increase this to $1.5 \mathrm{sec}$. The input coupling network is external, and its time constant may be as large as is desirable. Since the stray capacitance is small in these amplifiers, their frequency response is reasonably good (up to $5-10 \mathrm{kc} / \mathrm{sec}$.). If the input impedance exceeds about $\frac{1}{2} \mathrm{meg}$, there is too much coupling between input and output, and there is a tendency to oscillate. In high resistance circuits a small condenser is shunted across the input resistor. It is believed that production models ${ }^{9}$ of this amplifier will have better shielding of input and output and will not be subject to this difficulty.

The input noise level of these amplifiers when tested with a low impedance signal varies from less than one microvolt to ten or more. The equivalent noise resistance of the best amplifiers is several thousand ohms, and the average is roughly $20 \mathrm{~K}$. In the interests of economy of space the plate and cathode resistors of the first tube are composition, not wire. Where the above noise figures are not good enough, an especially selected low noise tube and wire-wound components may be used as a preamplifier. The same values of grid current as were obtained with the d.c. amplifier obtain here (less than $0.01 \mu \mathrm{a}$ ) while special selection gives considerably lower currents.

\subsection{Applications}

There are numerous applications for these a.c. amplifiers, and an example of their use as a carrier amplifier is given below.

${ }^{9}$ Laboratory for Electronics, Boston, is believed to be preparing production designs for several types of these amplifiers. 


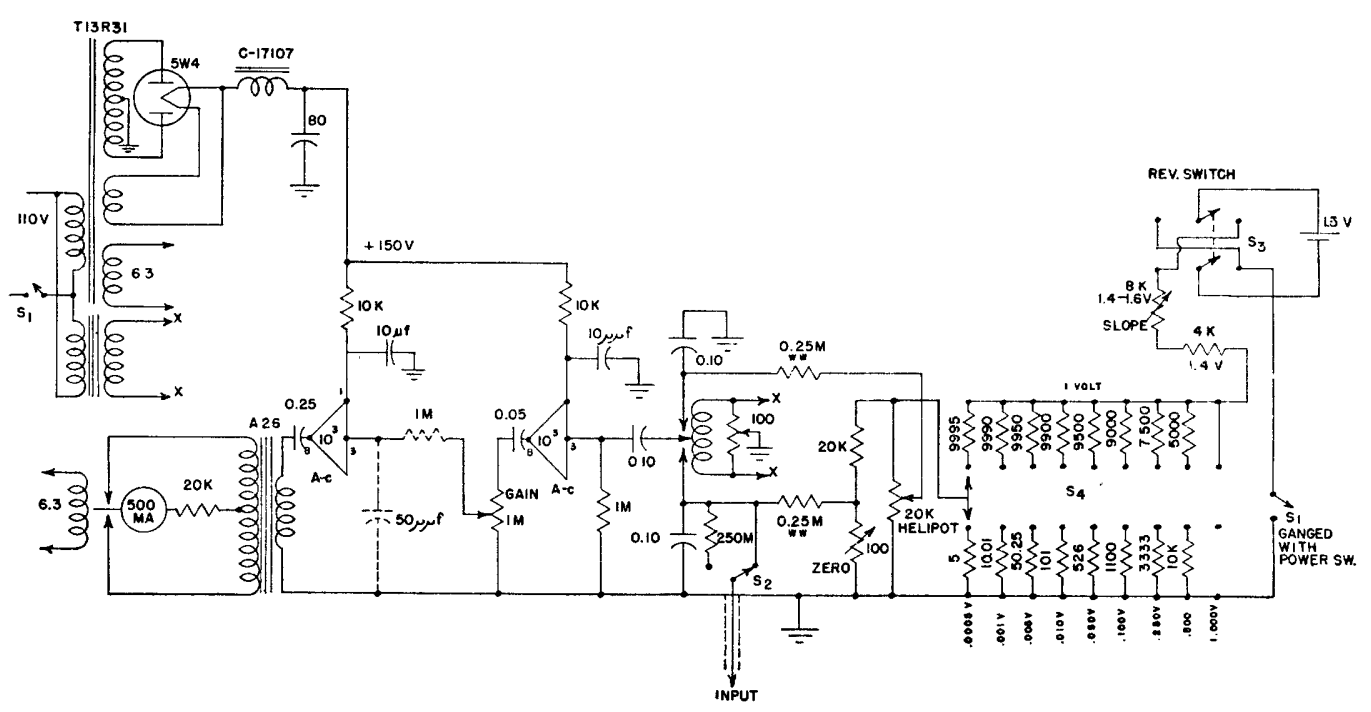

FIG. 6. Millivoltmeter circuit.

The use of mechanical-switch modulators for highly stable measurements in low resistance circuits was described recently, ${ }^{10}$ and here and elsewhere ${ }^{8}$ some designs for high-resistance circuits are given for the Brown Instrument Company's $\dagger$ "Converter."

Since the drift of these mechanical switches is much less than one thousandth that of thermionic amplifiers, ${ }^{8}$ a much more accurate and stable millivoltmeter can be constructed. Although this circuit is designed for simplicity and compactness, the performance of the circuits described in a companion paper ${ }^{8}$ can be obtained with a more bulky power supply. As shown, the circuit has a precision of 0.05 percent on the 1 volt scale limited by the linearity of the potentiometer.

The circuit of Fig. 6 uses a single-pole doublethrow mechanical switch to give full-wave modulation of the error between the input signal and a bucking-out voltage obtained from a precision potentiometer. The input voltage is connected either directly to the $0.25 \mathrm{M}$ input resistor or through a $250 M$ series resistor for larger input voltages. The bucking-out voltage has nine scales, from $500 \mu \mathrm{v}$ to 1 volt. The circuit diagram shows the calculated resistance values for the

${ }^{10} \mathrm{Max}$ D. Liston, G. E. Quinn, W. E. Sargeant, and G. G. Scott, "Contact modulated amplifier to replace sensitive suspension galvanometers," Rev. Sci. Inst. 17 [5], 194-198 (1946).

$\uparrow$ Philadelphia, Penna. various scales. The maximum voltage range of the meter is, of course, increased by one thousand when the $250 \mathrm{M}$ resistor is in series with the input voltage. The supply for the bucking-out voltage is obtained from a $1.5-\mathrm{v}$ battery through a calibrating resistor which sets the voltage supplied to the bucking-out network at one volt. This calibration may be performed, for example, by connecting the input to a standard cell and by adjusting the slope control. The zero point is set by a $100-o h m$ rheostat with the input opencircuited.

Since a rapid response is not required, the voltages at the contacts of the modulator are heavily by-passed. The contacts are, therefore, adjusted so that they do not short-circuit the inputs in the mid-position of the switch arm; for if they were short-circuited, the charge on the condensers would tend to equalize through the contacts of the switch.

The carrier pick-up may be adjusted to a minimum by adjusting the potentiometer center tap of the transformer exciting the modulator. That the pick-up is a minimum is easily determined by setting the gain control to zero, noting the output meter reading (it should be zero), and then noting that the output reading remains at zero while the gain is increased.

Since the a.c. amplifier units are mounted close together, the band width is narrowed by 


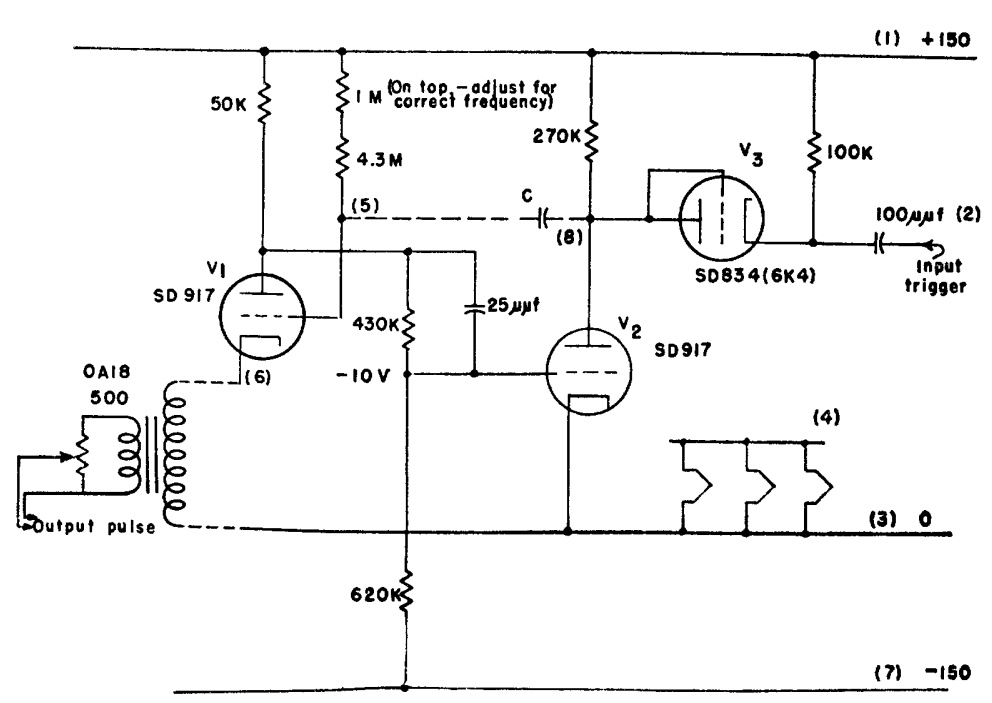

FIg. 7. Circuit of Monostablemultivibrator frequency divider. The timing condenser $C$ is mounted externally. an $R C$-filter to avoid oscillation. The output from the second amplifier is capacitance-coupled to a step-down transformer which also gives push-pull output suitable for full-wave demodulation by a second mechanical switch. The contacts of the demodulator are phased so as to have a duty ratio less than that of the modulating contacts. In this way small variations of the duty ratio of either modulator or demodulator will not affect the performance. The output voltage of \pm 3 volts is read on a $500-\mu$ a meter. The net gain is roughly $2 \times 10^{5}$, so that an error of several microvolts can be detected easily.

The limits to the performance of this instrument are set by the a.c. pick-up from the power supply and by the heater-to-cathode pick-up in the first amplifier tube. Depending upon the shielding of the power transformer and the bias applied to the heaters, this pick-up may vary from 30 to $300 \mu \mathrm{v}$, in the former case just satisfactory for measurements on the $500-\mu \mathrm{v}$ scale. Where operation at much lower levels is desired, the unit may be supplied externally, and direct current may be used for the heaters. Under these conditions only several microvolts pick-up will be observed. The limit to the measurement will be set by the thermal agitation noise in the input resistor and the amplifier noise, and the drift rate will be as low as $5 \times 10^{-9}$ volt $/$ minute. ${ }^{8}$

\section{MULTIVIBRATOR}

The multivibrator frequency divider lends itself to this type of packaging and Fig. 7 shows the package and its circuit diagram. The monostable multivibrator ${ }^{11}$ is triggered through a diode, and the output pulse is differentiated by a pulse transformer (formerly made by Utah-Type 0A18). The bias of the normally non-conducting tube $\left(V_{2}\right)$ is set at roughly $-10 \mathrm{v}$, since regeneration does not occur until a bias of roughly $-5 \mathrm{v}$ obtains. The trigger from a similar multivibrator appears as a pulse of roughly $+15 \mathrm{v}$ at the grid of $V_{2}$ and hence the triggering is reliable. The particular design was used to generate a series of timing markers at $20,10,5,1$, and $\frac{1}{5}$ c.p.s. The pulses from each frequency divider may be directly added in the secondaries of the pulse transformers.

\section{ACKNOWLEDGMENT}

The construction and early design work was due to Mr. A. E. Berg and Miss M. Celeste Babineau. Mr. E. F. Mac Nichol, Jr. designed the multivibrator circuit. Mrs. Rose Brogrow's assistance in checking the manuscript is gratefully acknowledged.

${ }^{11}$ For details of nomenclature and circuit design see Radiation Laboratory Series (McGraw-Hill Book Company, Inc., New York), 19, Chapter 5 (in press). 
